\title{
Gering erhöhtes Risiko intrakranieller Blutungen unter SSRI
}

Fragestellung: Systematisches Review und Metaanalyse über epidemiologische Studien zur Frage der Häufigkeit des Auftretens intrakranieller Blutungen unter antidepressiver Therapie mit selektiven Serotonin-Wiederaufnahme-Hemmern (SSRI).

Hintergrund: Aufgrund der plättchenaggregationshemmenden Eigenschaften von SSRI besteht ein erhöhtes Risiko für gastrointestinale Blutungen als unerwünschte Arzneimittelwirkung (UAW), insbesondere bei Komedikation mit gerinnungshemmenden Medikamenten. Eine Risikoerhöhung wird auch für intrakranielle Blutungen (insbesondere subdurale und intrazerebrale Hämatome) angenommen, doch bisherige Studien waren zum Teil widersprüchlich.

Patienten und Methodik: Kontrollierte prospektive Beobachtungsstudien wurden über gängige Datenbanken (Medline, Web of Sciences, EMBASE, Scopus, ProQuest) identifiziert und ausgewertet. Es wurden keine klinischen Therapiestudien (randomisierte kontrollierte Studien, RCT) berücksichtigt aufgrund

Hackam DG, Mrkobrada M. Selective serotonin reuptake inhibitors and brain hemorrhage: a meta-analysis. Neurology 2012; 79: $1862-5$ der Seltenheit der Berichte von intrakraniellen Blutungen im Rahmen der begrenzten Laufzeiten.

Ergebnisse: Insgesamt wurden 16 prospektive Studien als relevant und geeignet eingestuft und ausgewertet (elf FallKontrollstudien, vier Kohortenstudien, eine Fallstudie mit Crossover-Design). 14 Studien waren populationsbasiert, zwei Studien krankenhausbasiert. Intrakranielle Blutungen waren mit SSRI-Behandlung assoziiert und zwar sowohl in der nichtadjustierten (Rate Ratio [RR] 1,48, 95\%-Konfidenzintervall [KI] $1,22-1,78)$ als auch in der adjustierten Analyse (RR 1,51, 95\%KI 1,26-1,81), ebenso intrazerebrale Blutungen (nicht adjustiert: RR 1,68, 95\%-KI 1,46-1,91; adjustiert: RR 1,42, 95\%-KI 1,231,65). In fünf der untersuchten Studien (drei zu intrakraniellen Blutungen und je eine zu hämorrhagischem Schlaganfall und intrazerebraler Blutung) wurde eine Risikoerhöhung bei einer Kombinationstherapie mit SSRI plus oralen Antikoagulanzien im Vergleich zu einer Therapie ausschließlich mit oralen Antikoagulanzien gefunden (RR 1,56, 95\%-KI 1,33-1,83). Bei gepoolter Analyse aller Studien fand sich eine Risikoerhöhung bei allen Kohortenstudien (RR 1,61, 95\%-KI 1,04-2,51), Fall-Kontroll-Studien (Odds ratio [OR] 1,34, 95\%-KI 1,20-1,49) sowie Fallbeobachtungsstudien mit Crossover-Design (OR 4,24, $95 \%$ KI 1,95-9,24).

Schlussfolgerungen: Das Auftreten intrakranieller (ganz überwiegend intrazerebraler) Blutungen ist mit SSRI-Behandlung signifikant assoziiert, wobei allerdings aufgrund der Seltenheit dieser UAW in der Praxis ein eher geringes absolutes Risiko besteht.

\section{- Kommentar von Christian Lange-Asschenfeldt, Düsseldorf}

\section{Bei hohem Risiko andere Antidepressiva erwägen}

Die jährliche Inzidenz von Hirnblutungen wird von verschiedenen, nicht zuletzt ethnischen Faktoren beeinflusst und wurde weltweit mit 24,6 pro 100.000 Personen angegeben [1]. Auf der Basis dieser Zahl und ihren Ergebnissen errechnen die Autoren der vorliegenden Studie, dass unter SSRI-Therapie mit je einer zusätzlichen intrazerebralen Blutung pro 10.000 Personen gerechnet werden muss. Somit ergibt sich global ein sehr geringes absolutes Risiko trotz der klaren Assoziation. Als Limitation der vorliegenden Metaanalyse muss eine gewisse Heterogenität der untersuchten Studien, sowohl im Hinblick auf die Stichprobengrößen als auch bezüglich der Adustierungskriterien, angesehen werden. Die Autoren selbst schließen daraus, dass weitere Studien mit größeren Stichprobenumfängen nötig seien, in denen auch besser einzelne Subgruppen intrakranieller Blutungen abzugrenzen wären. Aufgrund dieser Studie sollte sicherlich keine allgemeine Änderung der generellen Verschreibungsgewohnheiten hinsichtlich SSRI erfolgen, jedoch sollte bei Patienten mit Risikofaktoren für intrakranielle Blutungen (vor allem orale Langzeit-Antikoagulation, frühere intrakranielle Blutung, zerebrale Amyloidangiopathie, schwere chronische Alkoholabhängigkeit, schwer kontrollierbare Hypertonie) eine sorgfältige Nutzen-Risiko-Abwägung erfolgen und gegebenenfalls Antidepressiva aus anderen Klassen zur Behandlung erwogen werden.

\section{Referenz \\ 1. Van Asch CJ et al. Lancet Neurol 2010; 9: 167-76}

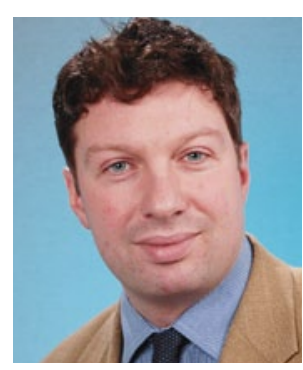

PD Dr. med. Christian Lange-

Asschenfeldt, Düsseldorf

Leitender Oberarzt Gerontopsychiatrie Facharzt für Neurologie, Psychiatrie und Psychotherapie Klinik und Poliklinik für Psychiatrie und Psychotherapie der Heinrich-Heine-Universität, LVR-Klinikum Düsseldorf. E-Mail: kn50050@lvr.de 